\title{
Intracellular gallium nitride microrod laser
}

\author{
Minho S. Song ${ }^{1}$, Hyeonjun Baek², Keundong Lee ${ }^{1}$, Dongha Yoo ${ }^{1}$, Kunook Chung ${ }^{3}$, Jamin Lee ${ }^{1}$, Hyungseok C. Moon , \\ Byung Hun Lee ${ }^{1}$, Hye Yoon Park (D) and Gyu-Chul Yi (i)
}

\begin{abstract}
We report laser emission from gallium nitride $(\mathrm{GaN})$ microrods that are introduced into mammalian cells and the application of these microrods for cell labeling. GaN microrods were grown on graphene-coated $\mathrm{SiO}_{2} / \mathrm{Si}$ substrates by metal-organic vapor phase epitaxy. The GaN microrods are easily detached from the substrates because of the weakness of the van der Waals forces between GaN and graphene. The uptake of microrods into HeLa cells via endocytosis and viability after uptake were investigated. Normal cellular activities, including migration and division, were observed over 2 weeks in culture. Furthermore, the photoluminescence spectra of the internalized microrods exhibited sharp laser emission peaks with a low lasing threshold of $270 \mathrm{~kW} / \mathrm{cm}^{2}$.
\end{abstract}

\section{Introduction}

Cell labeling techniques play an essential role in cell imaging and individual cell tracking ${ }^{1}$. In medical and biological science, cell labeling is used to study cell migration $^{2}$ and differentiation ${ }^{3}$, the dynamic behavior of cells ${ }^{4}$, and the progression of diseases, including the metastasis of cancer ${ }^{5}$. Fluorescent dyes have been widely used for cell labeling, but their broad emission spectrum has been an obstacle to labeling large numbers of cells ${ }^{6-8}$. In addition, issues such as the low efficiency and photobleaching of organic materials remain to be resolved ${ }^{8}$. A new approach using intracellular lasers has been proposed to address these issues ${ }^{9-12}$. The few hundred-fold narrower linewidth and higher signal-to-noise ratio of intracellular laser probes demonstrated the advantages of using intracellular lasers over fluorescent dyes for cell labeling and tracking applications. In particular, inorganic optical materials ${ }^{13-19}$ have been evaluated for intracellular laser application because they offer excellent lasing characteristics and chemical and mechanical stability ${ }^{20-24}$. For example, inorganic materials, including cadmium sulfide (CdS) nanowires ${ }^{22}$, aluminum gallium phosphide

\footnotetext{
Correspondence: Gyu-Chul Yi (gcyi@snu.ac.kr)

${ }^{1}$ Department of Physics and Astronomy and Institute of Applied Physics, Seoul National University, Seoul 08826, South Korea

${ }^{2}$ Institute for Photonics and Quantum Sciences, SUPA, Heriot-Watt University, Edinburgh EH14 4AS, UK
}

Full list of author information is available at the end of the article
(AlGaP) multiquantum well nanodisks ${ }^{21}$, and indium gallium arsenide phosphide (InGaAsP) nanodisks ${ }^{20}$, have been used for inorganic intracellular lasers to exploit their advantages. While the toxicity of CdS and InGaAsP is an obstacle to the bioapplication of these compounds and the toxicity of aluminum gallium indium phosphide (AlGaInP) is not well known ${ }^{25}$, gallium nitride $(\mathrm{GaN})$ is a well-known, nontoxic, biocompatible material ${ }^{26,27}$ with high refractive index and optical gain coefficient ${ }^{28}$. Here, we report the fabrication of $\mathrm{GaN}$ microrod lasers and their lasing characteristics for intracellular laser applications.

\section{Materials and methods \\ GaN microrod growth by CVD on graphene}

$\mathrm{GaN}$ microrods were grown on graphene films by metal-organic vapor phase epitaxy (MOVPE), as shown in Fig. 1a. Graphene films were synthesized on copper foil using chemical vapor deposition (CVD) and transferred onto supporting substrates of amorphous $\mathrm{SiO}_{2}$-coated $\mathrm{Si}$ $\left(\mathrm{SiO}_{2} / \mathrm{Si}\right)$. Typically, CVD-grown graphene films are semitransparent, multilayer graphene with electrical resistance in the range of $600-800 \Omega /$ sq. GaN microrods were grown on graphene films using a two-step temperature process: $750-850^{\circ} \mathrm{C}$ for $3 \mathrm{~min}$ and $950-1050{ }^{\circ} \mathrm{C}$ for $30 \mathrm{~min}$. The substrate was then heated at $1100^{\circ} \mathrm{C}$ for $10 \mathrm{~min}$ with hydrogen. Trimethyl gallium (TMGa), ditertiary butyl silane (DTBSi), and ammonia $\left(\mathrm{NH}_{3}\right)$ were 
employed as reactants to grow the $\mathrm{GaN}$ microrods, and nitrogen was used as the carrier gas. The flow rates of TMGa, DTBSi, and $\mathrm{NH}_{3}$ were in the ranges of $15-30$, $1-3$, and $100-500 \mathrm{sccm}$, respectively. The pressure of the reactor chamber was maintained at 300 Torr with a mixture of hydrogen and nitrogen gases during the process. The GaN microrods grown over the entire graphene film had a uniform areal density of $10^{7} \mathrm{~cm}^{-2}$.

\section{Cell culture}

HeLa cells were cultured in modified Eagle's medium supplemented with $10 \%$ fetal bovine serum, 1\% GlutaMAX, and $1 \%$ penicillin/streptomycin and maintained at $37^{\circ} \mathrm{C}$ in a humidified atmosphere with $5 \% \mathrm{CO}_{2}$.

\section{Internalization of GaN microrods into cells}

The as-grown GaN microrods on the graphene/ $/ \mathrm{SiO}_{2} / \mathrm{Si}$ substrate were sterilized in 95\% ethyl alcohol for $1 \mathrm{~h}$, and then, $\mathrm{GaN}$ microrods were detached from the substrate in $1 \mathrm{ml}$ of cell culture medium by sonication for $1 \mathrm{~min}$. The concentration of $\mathrm{GaN}$ microrods in the cell culture medium was adjusted to $10^{7} \mathrm{ml}^{-1}$, and then, $200 \mu \mathrm{l}$ was added to a cell culture dish using a micropipette. GaN microrods were naturally internalized into the cells via endocytosis a few hours after being added to the cell culture medium.

\section{Fluorescence imaging}

Calcein AM and 4'-6-diamidino-2-phenylindole (DAPI) were used to label the cytoplasm and nucleus, respectively. Fluorescence images were obtained using an inverted microscope (Olympus IX73) equipped with an electron-multiplying charge-coupled device (EMCCD) camera (Andor) and an XYZ automated stage (ASI). For epifluorescence excitation, cells were illuminated with a white LED (Lumencor) and visualized with a $\times 40 / 0.6$ NA objective (Olympus). Fluorescence signals were collected by the same objective and delivered to an EMCCD camera after passing through a dichroic mirror and appropriate filter sets (Chroma 49002 ET-EGFP filter set for calcein AM and 49000 ETDAPI filter set for DAPI).

\section{Optical setup for lasing experiments}

Lasing experiments were conducted with microphotoluminescence $(\mu-\mathrm{PL})$ spectroscopy at room temperature. The third harmonic $(355 \mathrm{~nm})$ of a Nd:YAG laser $(10 \mathrm{~Hz}, 10 \mathrm{~ns}$ pulse width) was used as the excitation source, and the laser beam was focused to a spot size of $8 \mu \mathrm{m}$ through a UV objective lens $(\mathrm{NA}=0.50)$. A conventional motorized stage was used to move the sample, and emitted lasing signals were analyzed with a conventional monochromator and a charge-coupled device (CCD) camera.

\section{Results and discussion}

Schematic illustrations of the experimental procedure are shown in Fig. 1a. We used the experimental method described in our previously reported conference paper ${ }^{29}$. $\mathrm{GaN}$ microrods were prepared on CVD-grown graphene films using MOVPE without metal catalysts ${ }^{30}$. As shown in Fig. 1b, the fabricated GaN microrods typically had a diameter of $1.5 \pm 1 \mu \mathrm{m}$ and a length of $10 \pm 3 \mu \mathrm{m}$. Microrods that were detached from the substrate using sonication were added to HeLa cell cultures, as shown in Fig. 1c. Optical microscopy (OM) revealed bright spots (indicated by arrows) corresponding to the GaN microrods. HeLa cells internalized the microrods by endocytosis within a few hours after the microrods were added to the culture medium. Intracellular lasing spectra were obtained by confocal $\mu$-PL spectroscopy.

We investigated the endocytosis of a microrod into a cell before the lasing experiments. Representative time-lapse images (Fig. 2a) show that a HeLa cell was initially migrating

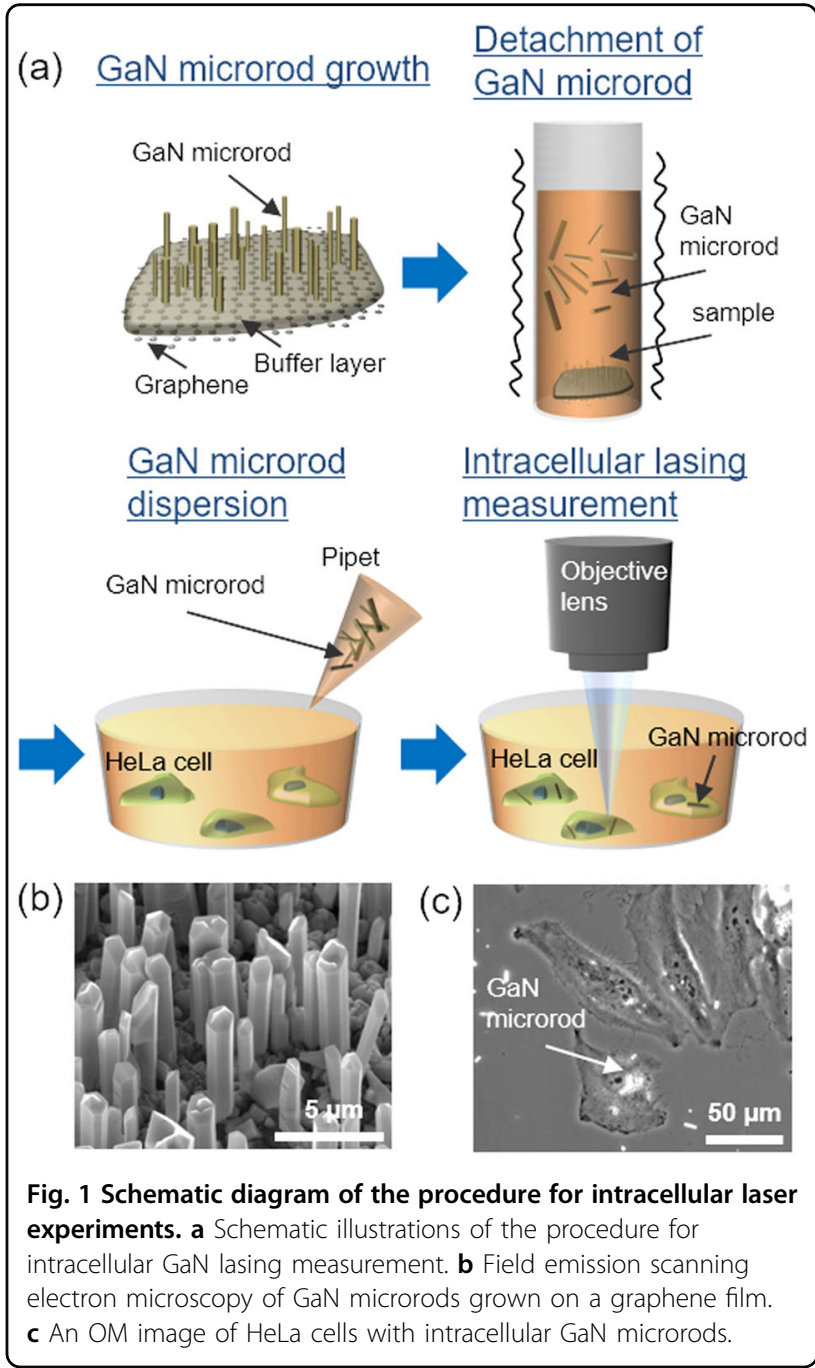


(a)

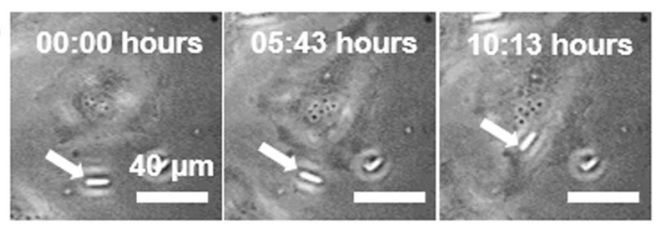

(b)
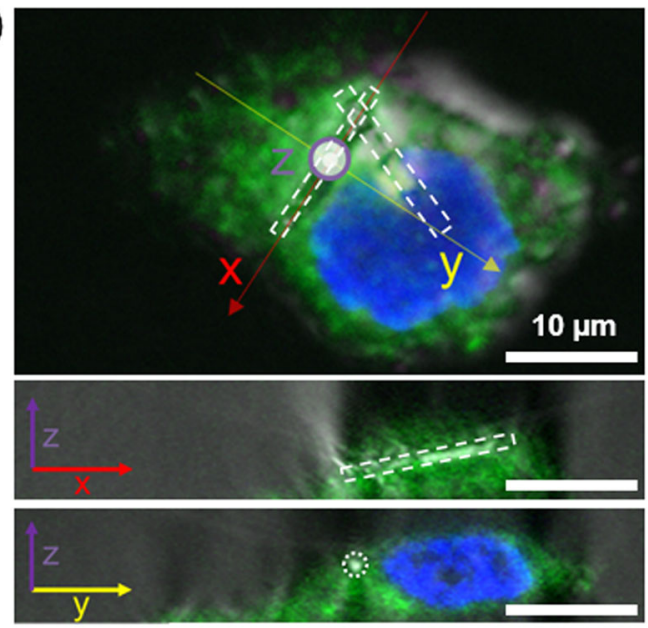

Fig. 2 Endocytosis of GaN microrod into HeLa cells. a Time-lapse images of the endocytosis of a GaN microrod into a HeLa cell. b Z-projection (top) of and cross-sectional (center and bottom) fluorescent CLSM images with maximum intensity. Blue and green indicate the nucleus and cytoplasm, respectively.

near a GaN microrod for a few hours. After $5 \mathrm{~h}$, filopodia and lamellipodia extended to the microrod, as indicated by the arrow in Fig. 2a. The GaN microrod was fully internalized into the cell within several minutes. We also evaluated the internalization percentage using OM images. We found that more than $70 \%$ of cells contained GaN microrods out of 53 cells (Fig. S1). Because the GaN microrods have a high aspect ratio, they can be more easily internalized into cells than the particles that have the same volume but a low aspect ratio $^{31,32}$. To confirm that GaN microrods are internalized, three-dimensional (3D) fluorescence images were obtained by confocal laser scanning microscopy (CLSM). As shown in Fig. 2b, the Z-projection and two cross-sectional images along the axial and radial directions show that GaN microrods (outlined with white dashed lines) are observed in the cytoplasm. These results indicate that the GaN microrods were fully internalized into the cytoplasm.

The cellular activity was monitored using phasecontrast optical microscopy (OM) to evaluate the biocompatibility of the $\mathrm{GaN}$ microrods. Figure 3a shows representative time-lapse images of cell migration and normal cell division over 2 weeks in culture. The HeLa cell that contained two GaN microrods in Fig. 3a migrated randomly. After $5 \mathrm{~h}$, the cell entered mitosis and appeared to round up. The daughter cells are positioned in the (a)
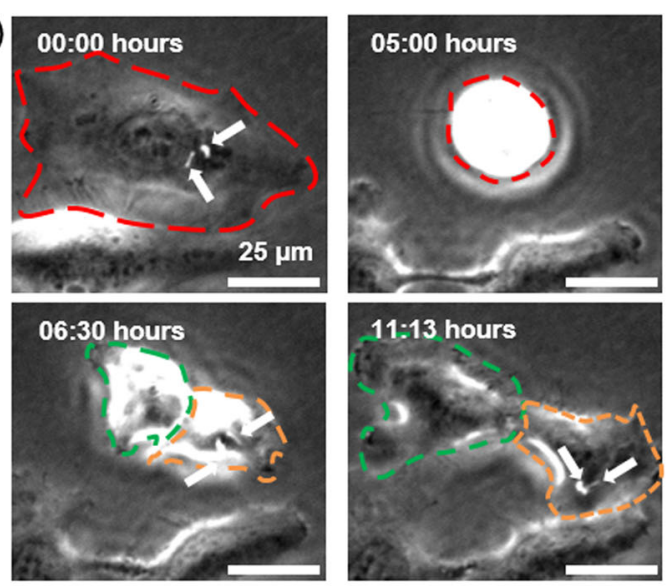

(b)
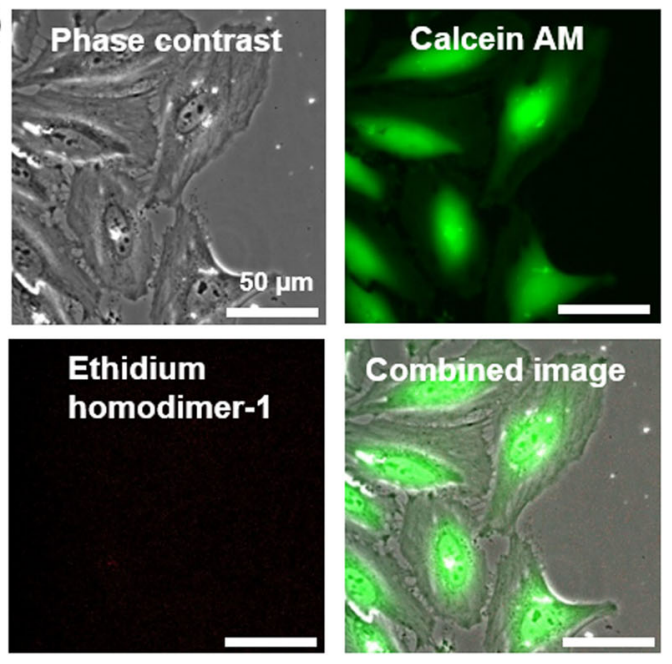

Fig. 3 Cell viability checking. a Time-lapse images of a HeLa cell undergoing normal cell division after internalizing a GaN microrod The cell indicated by the red dashed line divided into two cells indicated by the orange and green lines. b Phase-contrast and fluorescent optical microscopy images of HeLa cells. Fluorescent images were obtained using calcein AM and ethidium homodimer dyes.

adhesion pattern of the mother cell. This cell division appearance is consistent with normal cell division ${ }^{33}$. Following cell division, the internalized microrods (indicated by white arrows) remained in one of the daughter cells.

The cell viability was evaluated using a commercial assay. As shown in Figs. 3b and S2, fluorescence imaging of calcein acetoxymethyl (AM) was observed in all cells, indicating that they were viable. In contrast, no fluorescence was observed with ethidium homodimer staining in the same culture dish, indicating that no cells were dead. Superimposing the images from phase-contrast and calcein AM and ethidium homodimer fluorescence imaging confirmed that each cell imaged was viable.

$\mathrm{GaN}$ microrods in cells were investigated at room temperature using confocal $\mu$-PL spectroscopy. Figure 4a 
(a)

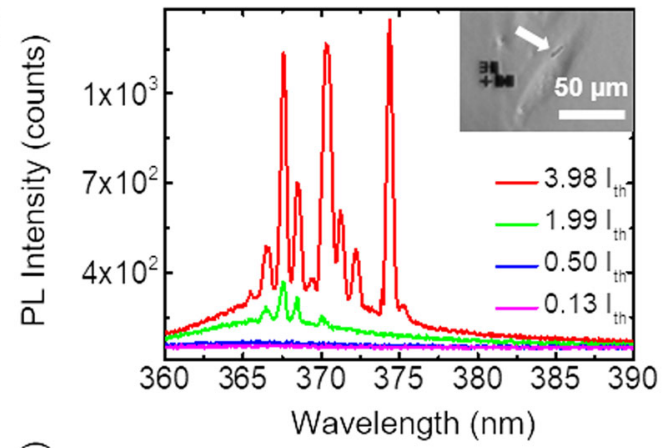

(b)

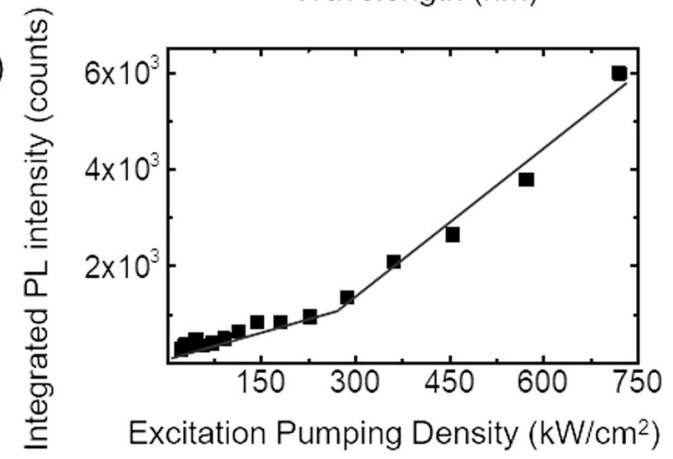

Fig. 4 Characteristics of intracellular GaN microrod laser. a PL spectra measured at different optical pumping power densities. The inset shows an OM image of the intracellular microrod used for the measurement. $\mathbf{b}$ Plot of output PL intensity versus input excitation power

shows the $\mu$-PL spectra of the GaN microrods at excitation power intensities ranging from 20 to $720 \mathrm{~kW} / \mathrm{cm}^{2}$. The inset shows an OM image of the GaN microrods used for these measurements. Below the lasing threshold $\left(I_{\text {th }}=\right.$ $270 \mathrm{~kW} / \mathrm{cm}^{2}$ ), a broad emission centered at $370 \mathrm{~nm}$ was observed around the near-band-edge (NBE) emission of $\mathrm{GaN}$. As the excitation power intensity increased, additional sharp peaks appeared in the NBE emission spectra and eventually became the dominant feature in the PL spectra. From the mode spacing, these sharp peaks were attributed to Fabry-Perot (FP)-type lasing along the axial direction of the microrods. According to previous reports of $\mathrm{GaN}$ microrod lasing on $\mathrm{SiO}_{2}$ substrates, the mode spacing for FP resonances is given by $\Delta \lambda \sim \frac{a}{L}$, where $\Delta \lambda$ is the mode spacing, $L$ is the cavity length, and $a=0.014$ $\mu \mathrm{m}^{2}$ is the value experimentally obtained by linear fitting between $\Delta \lambda$ and $1 / L^{23}$. For the lasing spectra shown in Fig. $4 \mathrm{a}, \Delta \lambda$ is approximately $1.11 \mathrm{~nm}$, which is in good agreement with the value calculated from the above equation when $L=12.3 \mu \mathrm{m}$, which is close to the length of the microrods. We also calculated the length dispersion of the GaN microrods from the mode spacing in Fig. S3. The average length of the $\mathrm{GaN}$ microrods is $11.5 \mu \mathrm{m}$ with a standard deviation of $2.4 \mu \mathrm{m}$. This calculated length dispersion of the GaN microrods from Fig. S3 is in good agreement with the length of the $\mathrm{GaN}$ microrods (a)

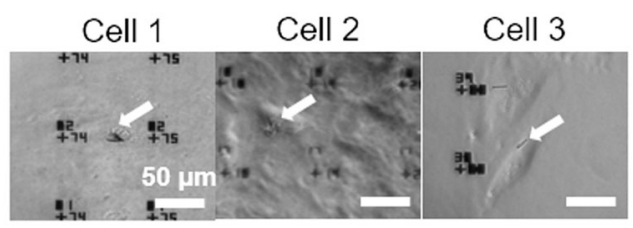

(b)

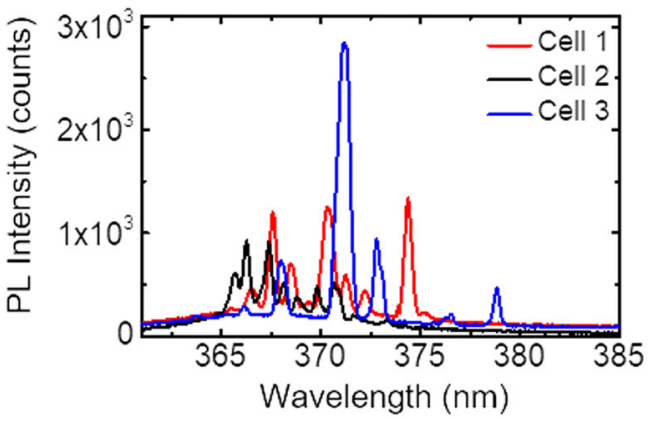

Fig. 5 Lasing spectra from different intracellular microrod lasers in living cells. a OM images of cells containing GaN microrods. Microrods are indicated by white arrows. $\mathbf{b}$ Lasing spectra obtained from the intracellular microrods shown in panel (a).

measured from the SEM image in Fig. 1b. Additionally, the measured $\Delta \lambda$ is substantially distinct from the WGM mode spacing estimated for the microrods, which is $\sim 10 \mathrm{~nm}$ for a microrod with a diameter of $1.5 \mu \mathrm{m}$, excluding the possibility of WGM lasing. Furthermore, the average Q-factor for the observed laser emission was estimated as 620 from the observed average linewidth of $0.6 \mathrm{~nm}$, which is a reasonable value for laser emissions from $\mathrm{GaN}$ microrods under intercellular conditions. The lasing spectra depend on the geometry of the microrods. Because the lasing exhibits Fabry-Perot type oscillation, the mode spacing depends on the length of the microrods. Additionally, the spectra could be influenced by the diameter, cross-sectional shape, or end-facet morphology. The diameters of the $\mathrm{GaN}$ microrods are larger than the single-mode diameter; therefore, transverse waveguide modes could be excited simultaneously in a single microrod. The existence of multiple transverse modes and competition between them resulted in complicated lasing spectra with irregular mode spacings and intensity ratios, as shown in Fig. 4a depicting a PL spectrum for an excitation power of $3.98 I_{\text {th }}$.

Figure $4 \mathrm{~b}$ shows a plot of the integrated PL intensity versus excitation pump intensity. At excitation power levels below the lasing threshold, the slope is 3.7 , but above the lasing threshold, the slope increases to 10.4 . The threshold value of $270 \mathrm{~kW} / \mathrm{cm}^{2}$ is similar to those reported for high-quality GaN micro- and nanostructures grown on single-crystal substrates ${ }^{34-36}$, demonstrating that the $\mathrm{GaN}$ microrods grown on graphene films are of high optical quality. Additionally, due to the relatively high refractive index of $\mathrm{GaN}$ microrods $\left(n_{\mathrm{GaN}}=2.6\right)^{37}$, the 
lasing threshold is low under intracellular conditions $\left(n_{\text {cell }}=1.36\right)^{38}$. The threshold value is also comparable to that in previously reported papers on inorganic intracellular lasers ${ }^{20,21}$. Because the $\mathrm{GaN}$ microrods have a comparable lasing threshold value along with nontoxicity and biocompatibility, $\mathrm{GaN}$ microrods can be candidates for intracellular laser applications.

We investigated the individual lasing characteristics of intracellular $\mathrm{GaN}$ microrod lasers. Figure $5 \mathrm{a}$, b show $\mathrm{OM}$ images of cells with internalized microrods and the corresponding lasing spectra, respectively. The lasing spectra from individual microrods are distinguishable by the center peak wavelength, mode spacings, and relative PL intensities between modes. In other words, each microrod has a distinct lasing spectrum, so each microrod can be tracked individually, making the GaN microrods suitable for cell labeling. Moreover, the effect of a refractive index change in the cell environment was similar for different transverse modes, suggesting that the profile of the laser emission spectra will not change substantially due to refractive index changes, as shown in Fig. S4. This property can be advantageous for cell tagging because the lasing spectra of individual microrods distinguished by multiple lasing modes can still be sustained under the condition of a refractive index change of the cell.

After conducting the lasing measurements, the cell viability was tested (Fig. S5). Even when HeLa cells were exposed to a focused laser beam at $10 \mathrm{~mJ} / \mathrm{cm}^{2}$ for $2 \mathrm{~s}$, the cells remained alive. Furthermore, cell nuclei were less exposed to the focused laser beam because the GaN microrods were typically positioned at a distance from the cell nucleus ${ }^{39,40}$.

\section{Conclusion}

In summary, we fabricated $\mathrm{GaN}$ microrod lasers for intracellular labeling applications. The $\mathrm{GaN}$ microrods grown on graphene films using MOVPE exhibited excellent lasing characteristics. The use of graphene as a substrate enabled facile detachment of the GaN microrods from the substrate and dispersion into culture media for internalization into cells. The high aspect ratio of the GaN microrods allowed them to be internalized into cells more easily. Excellent lasing signals were observed under intracellular conditions due to the optical quality and high refractive index of the GaN microrods. However, UV light can be inappropriate for long-term experiments and in vivo imaging. To avoid the use of a UV light source, our future plan is to fabricate an InGaN nanorod intracellular laser. By changing the In composition, we can tune the light emission up to the visible range. We believe that intracellular GaN-based microrod lasers can be candidates for cell labeling and tracking applications.

\section{Acknowledgements}

This work was supported by grants from the Global Research Laboratory Program (2015K1A1A2033332) and the Basic Science Research Program (2020R1A2C2007285) through the National Research Foundation of Korea (NRF). It was also supported by grants from the Research Fund (1.190103.01, 1.200046.01) of UNIST (Ulsan National Institute of Science \& Technology).

\section{Author details}

${ }^{1}$ Department of Physics and Astronomy and Institute of Applied Physics, Seoul National University, Seoul 08826, South Korea. ${ }^{2}$ Institute for Photonics and Quantum Sciences, SUPA, Heriot-Watt University, Edinburgh EH14 4AS, UK. ${ }^{3}$ Department of Physics, Ulsan National Institute of Science and Technology (UNIST), Ulsan 44919, South Korea

\section{Conflict of interest}

The authors declare no competing interests.

\section{Publisher's note}

Springer Nature remains neutral with regard to jurisdictional claims in published maps and institutional affiliations.

Supplementary information The online version contains supplementary material available at https://doi.org/10.1038/s41427-021-00299-8.

Received: 10 August 2020 Revised: 15 December 2020 Accepted: 11 February 2021.

Published online: 2 April 2021

\section{References}

1. Meijering, E., Dzyubachyk, O. \& Smal, I. Methods for cell and particle tracking. Method. Enzymol. 504, 183-200 (2012).

2. Denais, C. M. et al. Nuclear envelope rupture and repair during cancer cell migration. Science 352, 353-358 (2016).

3. Akhan, E., Tuncel, D. \& Akcali, K. C. Nanoparticle labeling of bone marrowderived rat mesenchymal stem cells: their use in differentiation and tracking. Biomed. Res. Int. 2015, 298430 (2015).

4. Ariel, G. et al. Swarming bacteria migrate by Levy Walk. Nat. Commun. $\mathbf{6}$, 8396 (2015).

5. van der Horst, G. et al. Real-time cancer cell tracking by bioluminescence in a preclinical model of human bladder cancer growth and metastasis. Eur. Urol. 60, 337-343 (2011)

6. Hill, M. T. \& Gather, M. C. Advances in small lasers. Nat. Photon. 8, 908-918 (2014).

7. McGloin, D. Cellular lasers. Nat. Photon. 9, 559-560 (2015).

8. Dean, K. M. \& Palmer, A. E. Advances in fluorescence labeling strategies for dynamic cellular imaging. Nat. Chem. Biol. 10, 512-523 (2014).

9. Schubert, M. et al. Lasing within live cells containing intracellular optical microresonators for barcode-type cell tagging and tracking. Nano Lett. 15, 5647-5652 (2015).

10. Humar, M. \& Yun, S. H. Intracellular microlasers. Nat. Photon. 9, 572-576 (2015).

11. Schubert, M. et al. Monitoring contractility in cardiac tissue with cellular resolution using biointegrated microlasers. Nat. Photon. 14, 452-458 (2020).

12. Humar, M. \& Yun, S. H. Whispering-gallery-mode emission from biological luminescent protein microcavity assemblies. Optica 4, 222-228 (2017).

13. Leong, E. S. P., Yu, S. F. \& Lau, S. P. Directional edge-emitting UV random laser diodes. Appl. Phys. Lett. 89, 221109 (2006).

14. Sun, X. W. et al. Room-temperature ultraviolet lasing from zinc oxide microtubes. Jpn J. Appl. Phys. 42, L1229-L1231 (2003).

15. Johnson, J. C. et al. Single gallium nitride nanowire lasers. Nat. Mater. 1, 106-110 (2002).

16. Arakawa, Y., Someya, T. \& Tachibana, K. Progress in GaN-based nanostructures for blue light emitting quantum dot lasers and vertical cavity surface emitting lasers. IEICE Trans. Electron. 83, 564-572 (2000).

17. Heilmann, M. et al. Vertically oriented growth of GaN nanorods on $\mathrm{Si}$ using graphene as an atomically thin buffer layer. Nano Lett. 16, 3524-3532 (2016). 
18. Choi, J. E., Yoo, J., Lee, D., Hong, Y. J. \& Fukui, T. Crystal-phase intergradation in InAs nanostructures grown by van der Waals heteroepitaxy on graphene. Appl. Phys. Lett. 112, 142101 (2018).

19. Dayeh, S. A., Yu, E. T. \& Wang, D. III-V nanowire growth mechanism: V/III ratio and temperature effects. Nano Lett. 7, 2486-2490 (2007).

20. Martino, N. et al. Wavelength-encoded laser particles for massively multiplexed cell tagging. Nat. Photon. 13, 720-727 (2019).

21. Fikouras, A. H. et al. Non-obstructive intracellular nanolasers. Nat. Commun. 9 , 4817 (2018)

22. Wu, X. Q. et al. Nanowire lasers as intracellular probes. Nanoscale $\mathbf{1 0}$ 9729-9735 (2018).

23. Baek, H., Hyun, J. K., Chung, K., Oh, H. \& Yi, G. C. Selective excitation of FabryPerot or whispering-gallery mode-type lasing in GaN microrods. Appl. Phys. Lett. 105, 201108 (2014).

24. Burgess, T. et al. Doping-enhanced radiative efficiency enables lasing in unpassivated GaAs nanowires. Nat. Commun. 7, 11927 (2016).

25. Liu, L. et al. Genotoxicity and cytotoxicity of cadmium sulfide nanomaterials to mice: comparison between nanorods and nanodots. Environ. Eng. Sci. 31, 373-380 (2014)

26. Hofstetter, M. et al. In vitro bio-functionality of gallium nitride sensors for radiation biophysics. Biochem. Biophys. Res. Commun. 424, 348-353 (2012).

27. Chen, C. R. \& Young, T. H. The effect of gallium nitride on long-term of neuritic function in cerebellar culture induced aging granule cells. Biomaterials 29, 1573-1582 (2008)

28. Shaklee, K. L., Nahory, R. E. \& Leheny, R. F. Optical gain in semiconductors. J. Lumin. 7, 284-309 (1973).
29. Song, M. S., Baek, H. \& Yi, G.-C. Intracellular GaN microrod laser. CLEO: Science and Innovations (Optical Society of America, 2019).

30. Chung, K. et al. Growth and characterizations of GaN micro-rods on graphene films for flexible light emitting diodes. APL Mater. 2, 092512 (2014).

31. Gratton, S. E. A. et al. The effect of particle design on cellular internalization pathways. Proc. Natl Acad. Sci. USA 105, 11613-11618 (2008).

32. Champion, J. A. \& Mitragotri, S. Role of target geometry in phagocytosis. Proc. Natl Acad. Sci. USA 103, 4930-4934 (2006).

33. Thery, M. \& Bornens, M. Cell shape and cell division. Curr. Opin. Cell Biol. 18 648-657 (2006).

34. Gradecak, S., Qian, F., Li, Y., Park, H. G. \& Lieber, C. M. GaN nanowire lasers with low lasing thresholds. Appl. Phys. Lett. 87, 173111 (2005).

35. Li, Q. M. et al. Single-mode GaN nanowire lasers. Opt. Express 20, 17873-17879 (2012).

36. $\mathrm{Xu}, \mathrm{H}$. W. et al. Gold substrate-induced single-mode lasing of GaN nanowires. Appl. Phys. Lett. 101, 221114 (2012).

37. Ejder, E. Refractive index of GaN. Phys. Status Solidi 6, 445 (1971).

38. Liu, P. Y. et al. Cell refractive index for cell biology and disease diagnosis: past, present and future. Lab Chip 16, 634-644 (2016).

39. Wong, D. Y., Ranganath, T., Kasko, A. M., Low-Dose \& Long-Wave, U. V. Light does not affect gene expression of human mesenchymal stem cells. PLOS ONE 10, e0139307 (2015)

40. Caricchio, R., McPhie, L. \& Cohen, P. L. Ultraviolet B radiation-induced cell death: critical role of ultraviolet dose in inflammation and lupus autoantigen redistribution. J. Immunol. 171, 5778-5786 (2003). 